\title{
Estudio comparativo de la nitrocarburación de los aceros AISI 4340 y AISI 347 mediante el proceso Tenifer-QPQ ${ }^{\circledR}$
}

\author{
Leornado Bellas ${ }^{\mathrm{a}}$, Gemma Castro ${ }^{\mathrm{b}}$, Laura Mera $^{\mathrm{b}}$, José L. Mier ${ }^{\mathrm{c}, \bigotimes}$, Ana García $^{\mathrm{c}}$, Angel Varela \\ ${ }^{a}$ Grupo Cetus, Polígono O Acevedo, Parcela, 15185 Cerceda, A Coruña, España \\ ${ }^{\mathrm{b}}$ Asociación de Investigación Metalúrgica del Noroeste (AIMEN), Relva St. 27, 36410 Porriño, Pontevedra, España \\ ${ }^{c}$ Escola Politécnica Superior de Ferrol, Universidade da Coruña, Rúa Mendizabal s/n, 15403 Ferrol, A Coruña, España \\ ( ${ }^{凶}$ Autor para la correspondencia: jose.mier@udc.es)
}

Enviado: 26 Febrero 2018; Aceptado: 2 Octubre 2018; Publicado on-Line: 26 Marzo 2019

\begin{abstract}
RESUMEN: Este trabajo estudia las diferencias microestructurales y tribológicas de las capas formadas durante la nitrocarburación ferrítica del acero aleado AISI 4340 y la nitrocarburación austenítica del acero inoxidable estabilizado AISI 347. Las muestras se sometieron a distintos tiempos de inmersión en un baño de nitrocarburación $(60,75,90,105$ y $120 \mathrm{~min})$ a $580^{\circ} \mathrm{C}$. Posteriormente se sometieron a un proceso de oxidación a $480{ }^{\circ} \mathrm{C}$ para formar una capa de $\mathrm{Fe}_{3} \mathrm{O}_{4}$. Los estudios de la microestructura de la capa nitrocarburada se realizaron por microscopía electrónica de barrido (SEM), espectroscopía de dispersión de energías (EDS) y difracción de rayos $\mathrm{X}$ (XRD). Se estudió el desgaste y el coeficiente de fricción de las muestras nitrocarburadas y las muestras no tratadas mediante el ensayo pink-on disk. Los resultados muestran tres zonas bien diferenciadas en el acero AISI 4340: una capa de óxidos externa, una capa blanca o de combinación y zona de difusión. Sin embargo, no se detectó la presencia de la capa de combinación en el acero AISI 347. En ambos aceros, el coeficiente específico de desgaste (k) de las muestras nitrocarburadas fue aproximadamente treinta veces menor que el de las muestras de referencia.
\end{abstract}

PALABRAS-CLAVE: Desgaste; Microestructura; Nitrocarburación austenítica; Nitrocarburación ferrítica; Nitrocarburación líquida; Tennifer ${ }^{\circledR Q P Q}$

Citar como/Citation: Bellas, L.; Castro, G.; Mera, L.; Mier, J.L.; García, A.; Varela, A. (2019). "Estudio comparativo de la nitrocarburación de los aceros AISI 4340 y AISI 347 mediante el proceso Tenifer-QPQ ${ }^{\circledR}$ ". Rev. Metal. 55(1): e136. https://doi.org/10.3989/revmetalm.136

\begin{abstract}
Comparative study of AISI 4340 and AISI 347 nitrocarburizing by Tenifer-QPQ® process. This paper studies the microstructural and tribological differences of layers formed during the ferritic nitrocarburizing of AISI 4340 alloy steel and AISI 347 stabilized stainless steel. The samples were exposed to different times of immersion in a nitrocarburizing bath $(60,75,90,105$ and $120 \mathrm{~min})$ at $580{ }^{\circ} \mathrm{C}$. Subsequently, they were subjected to an oxidation process at $480^{\circ} \mathrm{C}$ in order to form a $\mathrm{Fe}_{3} \mathrm{O}_{4}$ layer. Surface microstructural studies were carried out by SEM-EDS and x-ray diffraction (XRD). Wear and friction coefficient of nitrocarburized samples and non-treated samples were studied by pin-on-disk test. The results show two well-differentiated zones in AISI 4340 steel: an outer oxides layer, a white layer or compound layer and a diffusion zone. However, the compound layer was not found in AISI 347 steel. In both steels, the specific wear coefficient (k) of nitrocarburated samples is about thirty times lower than the reference samples.
\end{abstract}

KEYWORDS: Austenitic nitrocarburizing; Ferritic nitrocarburizing; Liquid nitrocarburizing; Microestructure; Tennifer®QPQ process; Wear

ORCID: Leonardo Bellas (https://orcid.org/0000-0002-1366-7758); Gemma Castro (https://orcid.org/0000-0003-26375348); Laura Mera (https://orcid.org/0000-0002-5533-533X); José L. Mier (https://orcid.org/0000-0003-2539-7684); Ana García (https://orcid.org/0000-0003-2969-1901); Angel Varela (https://orcid.org/0000-0002-1211-115X)

Copyright: (C) 2019 CSIC. Este es un artículo de acceso abierto distribuido bajo los términos de la licencia de uso y distribución Creative Commons Reconocimiento 4.0 Internacional (CC BY 4.0). 


\section{INTRODUCCIÓN}

La nitrocarburación en baño de sales TeniferQPQ ${ }^{\circledR}$ se ha utilizado por una gran variedad de industrias durante las últimas décadas para mejorar la resistencia al desgaste, corrosión y fatiga de piezas de acero y fundición. El tratamiento se realiza a temperaturas comprendidas entre 480 y $620^{\circ} \mathrm{C}$ en atmósfera oxidante con inmersión de la pieza en un baño de sales fundidas cuyo componente activo son cianatos alcalinos. Las reacciones que tienen lugar son:

Oxidación de cianuros:

$2 \mathrm{NaCN}(\mathrm{s})+\mathrm{O}_{2}(\mathrm{~g}) \rightarrow 2 \mathrm{NaCNO}(\mathrm{s})$

Descomposición del cianato:

$4 \mathrm{NaCNO}(\mathrm{s}) \rightarrow \mathrm{Na}_{2} \mathrm{CO}_{3}(\mathrm{~s})+2 \mathrm{NaCN}(\mathrm{s})+$ $\mathrm{CO}(\mathrm{g})+2 \mathrm{~N}(\mathrm{~g})$

Se forma así nitrógeno atómico que se introduce en el metal por difusión. Mientras, el monóxido de carbono se disocia sobre la superficie del acero a través de la reacción:

$$
2 \mathrm{CO}(\mathrm{g}) \rightarrow \mathrm{C}(\mathrm{s})+\mathrm{CO}_{2}(\mathrm{~g})
$$

generando carbono que también se difunde al interior del acero.

Además este proceso incluye dos etapas de oxidación que tienen como objeto la creación de una capa de magnetita $\left(\mathrm{Fe}_{3} \mathrm{O}_{4}\right)$ la cual aumenta la resistencia a la corrosión y al desgaste, aunque todavía no se conoce el mecanismo por el cual esto sucede (Marušić et al., 2006; Li et al., 2008a), y una etapa de pulido intercalada entre las anteriores para disminuir la rugosidad de las superficies nitrocarburadas.

En los aceros al carbono (AISI 10xx) y los aceros aleados Cr-Ni-Mo (AISI 4xxx) la nitrocarburación ferrítica (FNC) se suele realizar a temperaturas entre 500 y $590{ }^{\circ} \mathrm{C}$, mientras que la nitrocarburación austenítica (ACN) se lleva a cabo entre $590{ }^{\circ} \mathrm{C}$ y $700{ }^{\circ} \mathrm{C}$ (Fattah y Mahboubi, 2010; Schneider, 2014). En ambos casos se forma una capa externa, capa de compuestos, que da al material resistencia a la corrosión y al desgaste, y una zona interna de difusión que aumenta la resistencia a la fatiga del acero (Mittemeijer, 2013; Brühl et al., 2016). En la capa de compuestos tiene lugar la precipitación de nitruros y carbonitruros de hierro como la fase $\varepsilon$ $\left(\mathrm{Fe}_{2-3}(\mathrm{C}, \mathrm{N})\right)$ y fase $\gamma^{\prime}\left(\mathrm{Fe}_{4}(\mathrm{C}, \mathrm{N})\right)$ y nitruros de elementos de aleación. Las diferencias microestructurales más significativas entre un tipo de proceso y otro son dos: por una parte, en la nitrocarburación austenítica tiene lugar la aparición de la subcapa $\gamma$ o fase $\mathrm{S}$ enriquecida en nitrógeno y carbono entre la capa de compuestos y la capa de difusión en la cual la red cristalina de la austenita sufre una expansión, y, por otra, el menor espesor de la capa de difusión en la nitrocarburación ferrítica que en la austenítica (Dong, 2010; Fattah y Mahboubi, 2010).

La existencia de austenita a temperaturas mucho más bajas en los aceros inoxidables austeníticos que los aceros AISI 1 xxx y AISI 4xxx posibilita la realización del proceso de nitrocarburación austenítica a las mismas temperaturas que la nitrocarburación ferrítica (Li et al., 2008a; Wang et al., 2014). Hay que tener en cuenta que en los aceros inoxidables austeníticos existen dos causas que pueden disminuir la cinética de difusión del nitrógeno y carbono en la matriz austenítica: en primer lugar, la existencia de la capa pasiva de $\mathrm{Cr}_{2} \mathrm{O}_{3}$ que hace de barrera difusional de dichos elementos intersticiales, $\mathrm{y}$, en segundo lugar, la gran cantidad de elementos de aleación que contienen estos aceros (Funatani, 2004; Flodström, 2012).

El presente trabajo tiene como objetivo establecer las diferencias más significativas de comportamiento frente al desgaste y su relación con las características mecánicas y microestructurales de los aceros AISI 4340 y AISI 347 nitrocarburados en baño de sales. El primero de ellos es un acero de baja aleación que, normalmente, se le suele emplear en nitrocarburación ferrítica, y el segundo se trata de un acero inoxidable austenítico con altos contenidos de cromo y níquel y la presencia de niobio como elemento estabilizante frente al fenómeno de sensibilización. Por último, este trabajo trata de profundizar en el conocimiento del proceso nitrocarburación en baño de sales de aceros inoxidables estabilizados sobre los que apenas se han realizados estudios.

\section{MATERIALES Y MÉTODOS}

La primera etapa del proceso Tenifer-QPQ ${ }^{\circledR}$ consistió en un precalentamiento en aire a $420{ }^{\circ} \mathrm{C}$ de 20 a $40 \mathrm{~min}$. En la segunda etapa las piezas se introdujeron en un baño que contenía una mezcla de cianuros y carbonatos alcalinos a $580{ }^{\circ} \mathrm{C}$ creándose, de esta manera, la capa nitrocarburada. Los tiempos de nitrocarburación empleados fueron 60 , $75,90,105$ y $120 \mathrm{~min}$. En la siguiente, las probetas se metieron en un baño de nitritos y nitratos a $420^{\circ} \mathrm{C}$ durante $15 \mathrm{~min}$ en el que se produce la oxidación superficial del acero que origina $\mathrm{Fe}_{3} \mathrm{O}_{4}$. En la cuarta etapa se realiza el pulido de las muestras a temperatura ambiente con chorreado de perlas de vidrio de 40-70 $\mu \mathrm{m}$ de diámetro a 3 bares de presión. En la última etapa se realiza un nuevo tratamiento de oxidación a $420^{\circ} \mathrm{C}$ durante $25 \mathrm{~min}$.

Para este estudio se emplearon el acero AISI $4340 \quad(0,29 \% \mathrm{C}, \quad 0,31 \% \mathrm{Si}, \quad 0,75 \% \mathrm{Mn}, \quad 2,12 \% \mathrm{Cr}$, $0,42 \% \mathrm{Mo}, 1,99 \% \mathrm{Ni},<0,025 \% \mathrm{P},<0,035 \% \mathrm{~S}$, resto $\mathrm{Fe})$ 
templado en aceite desde $800{ }^{\circ} \mathrm{C}$ y revenido a $600{ }^{\circ} \mathrm{C}$ durante $2 \mathrm{~h}$, y el acero inoxidable AISI $347(0,05 \% \mathrm{C}$, $0,53 \% \mathrm{Si}, 1,53 \% \mathrm{Mn}, 17,41 \% \mathrm{Cr}, 9,28 \% \mathrm{Ni}, 0,42 \% \mathrm{Nb}$, $0,02 \% \mathrm{P},<0,009 \% \mathrm{~S}$, resto $\mathrm{Fe})$. Las composiciones se determinaron mediante un espectrómetro de chispa, modelo Spectromax LMF04 de Spectro. Tanto las muestras del acero AISI 4340 como las del AISI 347 se identificaron en función del tiempo de nitrocarburación: A-60, A-75, A-90, A-105 y A-120 para las muestras de acero AISI 4340 y S-60, S-75, S-90, S-105 y S-120 en el caso del acero AISI 347. La cifra indica el número de minutos en el baño de nitrocarburación.

Las secciones transversales de las capas nitrocarburadas se analizaron mediante microscopía óptica con un banco metalográfico Leica MEF4A y microscopía electrónica de barrido (SEM) con un equipo Hitachi S4800 equipado con un espectrómetro de energías dispersivas (EDS) Bruker Quantax 400. Se determinó la estequiometría de los compuestos de la capa blanca y capa de óxidos superficiales mediante difracción de RX con un difractómetro Siemens D5000 que utilizó la radiación $\mathrm{K} \alpha$ del cobre. Para ello, se prepararon y analizaron las muestras indicadas con las siguientes condiciones: paso de $0,050^{\circ}$, tiempo por paso 5 segundos y potencia $30 \mathrm{~mA}$ y $40 \mathrm{KV}$.

La evolución de las microdurezas con la profundidad en el acero AISI 4340 se determinó con un microdurómetro Shimadzu que empleó un indentador de diamante con geometría Vickers. En el caso del acero AISI 347, las medidas de microdureza se han realizado mediante un nanoindentador NanoTest 600 de MicroMaterials Ltd debido al pequeño espesor de la capa de nitrocarburación obtenido, especialmente para un tiempo de permanencia de $60 \mathrm{~min}$. Esta técnica permite hacer huellas de pequeño tamaño sin necesidad de realizar una inspección visual de las mismas. Sin embargo, hay que decir que, dadas las bajas cargas aplicadas en la nanoindentación ( $30 \mathrm{mN}$ en el presente trabajo), existe una mayor dispersión de valores ya que son más sensibles a la presencia de heterogeneidades de la muestra. En el caso del acero AISI 4340 se realizó un barrido en cada muestra con 8 huellas, mientras que en el acero AISI 347 se realizó un barrido en cada muestra con un promedio de 7 a 11 huellas en la capa de nitrocarburación, y de 5 huellas en el material base. Para la determinación de la resistencia al desgaste se empleó un tribómetro MicroTest modelo MT2/60/SCM. Las capas superficiales objeto de estudio fueron sometidas a contacto deslizante mediante una configuración pin-on-disk, donde la contraparte estática (pin) se corresponde con una bola de alúmina (1500-1600 HV) con una carga normal de $10 \mathrm{~N}$ en la determinación de la resistencia al desgaste con objeto de aumentar las características abrasivas del ensayo, y acero de calidad AISI 52100 (600-800 HV) con una carga normal de $1 \mathrm{~N}$ en la determinación del coeficiente de fricción. La temperatura de ensayo fue $25^{\circ} \mathrm{C}$ y la distancia recorrida $250 \mathrm{~m}$. Hay que señalar que cada uno de estos ensayos se ha realizado tres veces con cada contraparte estática para asegurar la reproducibilidad de los resultados obtenidos. El volumen desgastado $(\Delta \mathrm{V})$ se ha calculado mediante perfilometría de contacto, y los valores de fuerza normal aplicada en el contacto y distancia de deslizamiento son $10 \mathrm{~N}$ y $500 \mathrm{~m}$ respectivamente.

\section{RESULTADOS Y DISCUSIÓN}

\subsection{Caracterización microestructural}

Como se puede ver en la Tabla 1, tanto el espesor de la capa de óxidos como el de la capa de nitruración aumentan con el tiempo de tratamiento de los dos tipos de aceros. En el acero AISI 4340 se alcanzan 4,7 $\mu \mathrm{m}$ en la capa de óxidos y 186,6 $\mu \mathrm{m}$ en la capa de nitrocarburación a los 120 min de tratamiento. Mientras, en el acero AISI 347 puede verse que el espesor de la capa de óxido alcanza en la probeta S-60 un espesor medio del orden de 1,2 $\mu \mathrm{m}$ y de $2,7 \mu \mathrm{m}$ en la probeta $\mathrm{S}-120$. En cuanto a los espesores de la capa nitrocarburada se sitúan entre 29 y $50 \mu \mathrm{m}$. Estos valores son mucho menores que los obtenidos en el caso del acero AISI 4340 con tiempos semejantes lo que se debe al alto contenido en cromo del AISI 347, que dificulta la difusión del nitrógeno y aumenta la cantidad de nitruros precipitados (Wu et al., 2015)

La microestructura obtenida en todas las muestras del acero AISI 4340 nitrocarburado (Fig. 1a) es muy similar: en la parte más externa, se observa una capa de óxidos de poco espesor y, por debajo de ésta, una capa de combinación o capa blanca formada por

TABLA 1. Espesores de las capas de óxidos y nitrocarburación de los aceros AISI 4340 y AISI 347 tras el tratamiento Tenifer-QPQ $\mathbb{R}$

\begin{tabular}{lcc}
\hline & \multicolumn{2}{c}{ Espesor $\mathbf{( \pm 0 , 5} \boldsymbol{\mu m})$} \\
\cline { 2 - 3 } Muestra & Capa de óxidos & $\begin{array}{c}\text { Capa de } \\
\text { nitrocarburación }\end{array}$ \\
\hline A-60 & 2,4 & 137,0 \\
A-75 & 1,9 & 152,2 \\
A-90 & 4,5 & 171,8 \\
A-105 & 3,5 & 175,0 \\
A-120 & 4,7 & 186,6 \\
S-60 & 1,2 & 29,5 \\
S-75 & 1,6 & 31,6 \\
S-90 & 1,8 & 33,7 \\
S-105 & 2,5 & 42,1 \\
S-120 & 2,7 & 50,5 \\
\hline
\end{tabular}



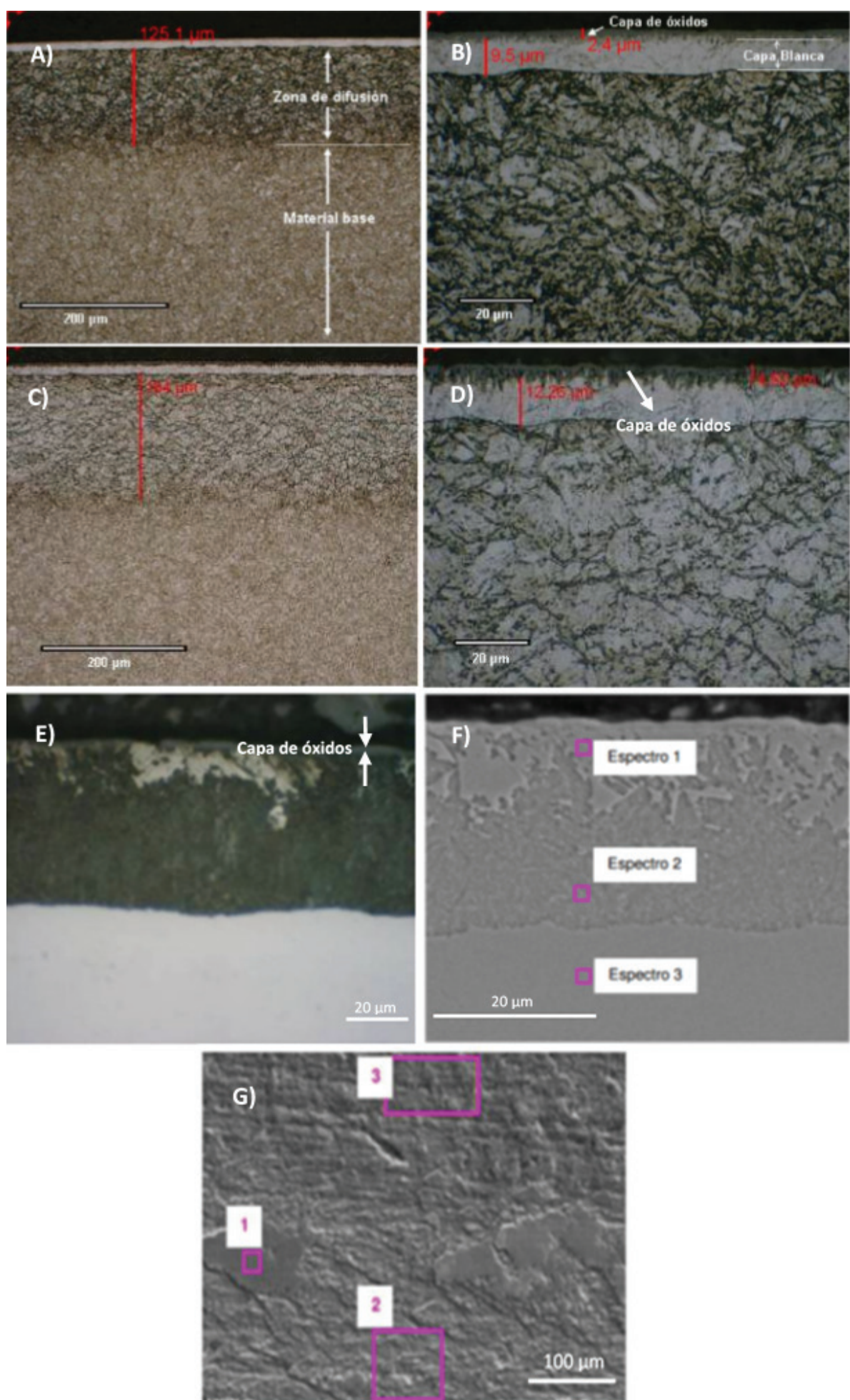

FIGURA 1. Imágenes SEM de las capas formadas durante la nitrocarburación de las muestra A-60 (a), A-60 a mayores aumentos (b), A-120 (c), A-120 a mayores aumentos (d), S-120 (e), sección transversal de la muestra S-60 junto con los puntos de análisis EDS (f), e imagen de la huella de desgaste en la muestra S-60 junto con los puntos de análisis EDS (g). 
carbonitruros de hierro y, posteriormente, una zona de difusión. El material base está constituido por martensita revenida. Por su parte, la observación por microscopía óptica de las muestras del acero AISI 347 (Fig. 1b) muestra una capa superficial de reducido espesor que se corresponde con el óxido formado y una capa de difusión, pero, a diferencia de lo que ocurre en el acero AISI 4340, no se distingue la presencia de capa blanca previa a la fase austenítica que forma el metal base.

Los análisis realizados mediante microscopía electrónica de barrido (SEM) y espectrometría de energías dispersivas (EDS) en las probetas nitrocarburadas durante 60 min de AISI 4340 (Fig. 2a) muestran que en la parte más externa del corte transversal hay una capa, de un espesor que varía aproximadamente entre 1,5 y 4,5 $\mu \mathrm{m}$, en la que la concentración de oxígeno es muy elevada. Este resultado confirma las observaciones realizadas mediante microscopía óptica donde la capa de óxidos no se puede ver tan claramente, debido a su pequeño espesor. Se observa también, la presencia de nitrógeno y hierro en dicha capa, aunque en mucha menor proporción que el oxígeno. En la zona de combinación existe una disminución drástica de la concentración de oxígeno que, luego, se mantiene prácticamente constante hasta la profundidad final analizada, mientras que el contenido de nitrógeno presenta un máximo a en torno a $5 \mu \mathrm{m}$ para, posteriormente, disminuir ligeramente y mantenerse prácticamente constante en la parte final del espectro que se corresponde con la zona de difusión. La concentración de hierro se mantiene prácticamente constante en un valor muy elevado a partir de $3 \mu \mathrm{m}$ ya que es el elemento mayoritario tanto de la capa de combinación como de la zona de difusión.

Los análisis mediante microscopía electrónica de barrido EDS del acero AISI 347 nitrocarburado durante $60 \mathrm{~min}$ (Fig. 2a) mostraron una alta concentración de oxígeno entre 1 y $3 \mu \mathrm{m}$ de profundidad que se corresponde con la formación de la capa de óxidos cuyo crecimiento de los óxidos prácticamente paralelo a la superficie de la aleación. Esto difiere de lo encontrado en el acero AISI 4340, en el que se apreciaban pequeños frentes de óxido perpendiculares a la superficie, lo que repercute, en cierta medida, en la adhesión entre las dos capas. Dentro de la capa de nitruración del acero AISI 347 se distingue una primera región de coloración clara y debajo de ella una capa de coloración más oscura y menos resistente al ataque del ácido empleado para revelarla (nital al 1\%). Esta observación coincide a la registrada por otros autores para la nitrocarburación por el proceso QPQ en aceros inoxidables endurecibles por precipitación (Li et al., 2008b). Aparentemente el tiempo de tratamiento parece influir no sólo en el espesor total de la capa nitrurada, sino también en una mayor presencia esta zona oscura. Debido a estas diferencias microestructurales se realizó un análisis detallado de la composición de dichas capas (Tabla 2). En él se aprecia que la región de coloración más oscura (espectro 2 de la Fig. 1f) tiene un
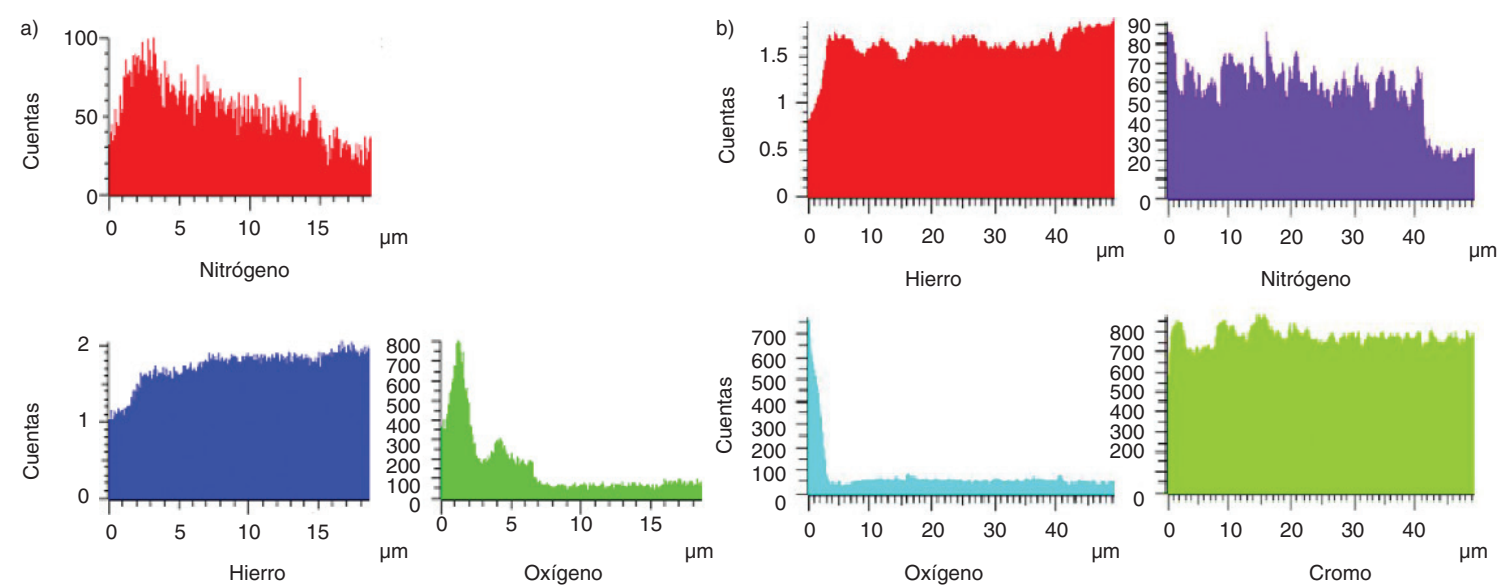

FIGURA 2. Análisis EDS en el que se muestra la variación con la profundidad de hierro, oxígeno y nitrógeno en la muestra A-60 (a) y cromo, hierro, oxígeno y nitrógeno en la muestra S-60 (b).

TABLA 2. Análisis de la composición química por EDS de tres regiones identificadas como Espectro 1, 2 y 3 de la Fig. 1f ( $\%$ peso).

\begin{tabular}{lccccccccc}
\hline Espectro & $\mathbf{C}$ & $\mathbf{N}$ & $\mathbf{O}$ & $\mathbf{S i}$ & $\mathbf{C r}$ & $\mathbf{M n}$ & $\mathbf{F e}$ & $\mathbf{N i}$ & $\mathbf{N b}$ \\
\hline Espectro 1 & 0,90 & 5,79 & 2,73 & 0,63 & 16,79 & 1,56 & 63,03 & 8,39 & 0,58 \\
Espectro 2 & - & 6,97 & 3,96 & 0,50 & 20,36 & 1,88 & 58,70 & 7,34 & 0,29 \\
Espectro 3 & 2,11 & - & 2,04 & 0,55 & 17,67 & 1,70 & 66,83 & 8,93 & 0,12 \\
\hline
\end{tabular}


mayor contenido en cromo y nitrógeno que la región más clara (espectro 1 de la Fig. 1f).

Los análisis de difracción de rayos $\mathrm{X}$ de la superficie de las muestras nitrocarburadas del acero AISI 4340 (Fig. 3a) mostraron, en todos los casos, picos correspondientes a magnetita $\left(\mathrm{Fe}_{3} \mathrm{O}_{4}\right)$ y a nitruros de hierro tipo $\varepsilon-\mathrm{Fe}_{2-3} \mathrm{~N}$ fundamentalmente, de manera similar a los resultados obtenidos por otros autores para la nitrocarburación de otros tipos de aceros inoxidables (García et al., 2018). Por otra parte, cabe destacar que es muy pequeña la cantidad encontrada de nitruro de hierro $\gamma^{\prime}-\mathrm{Fe}_{4} \mathrm{~N}$, nitruro de elevada fragilidad que, durante el proceso de post-oxidación, se ha transformado en $\mathrm{Fe}_{3} \mathrm{O}_{4}$ (Li et al., 2008b). Es importante que no se detecte $\mathrm{Fe}_{2} \mathrm{O}_{3}$ porque indicaría que se ha realizado correctamente la etapa de pulido intermedio entre el baño de nitrocarburación y el de oxidación cuyo objetivo principal es precisamente eliminar dicha capa de óxido ya que sus propiedades de resistencia frente a la corrosión no son deseables.

En cuanto a las fases detectadas en la superficie de las muestras nitrocarburadas del acero AISI 347 (Fig. 3b) se observó la presencia de: magnetita $\left(\mathrm{Fe}_{3} \mathrm{O}_{4}\right)$, nitruros de hierro $\left(\varepsilon-\mathrm{Fe}_{2-3} \mathrm{~N}\right.$ y $\left.\gamma^{\prime}-\mathrm{Fe}_{4} \mathrm{~N}\right)$, nitruro de cromo $\left(\mathrm{Cr}_{2} \mathrm{~N}\right)$ y carburo de cromo $\left(\mathrm{Cr}_{23} \mathrm{C}_{6}\right)$. La presencia de $\mathrm{Cr}_{2} \mathrm{~N}$ está relacionada con la descomposición de la austenita expandida o fase-S que es inestable a las temperaturas y tiempos alcanzados en el presente trabajo (Wang et al.,
2014). La presencia de $\gamma^{\prime}-\mathrm{Fe}_{4} \mathrm{~N}$ es más evidente en las muestras nitrocarburadas durante tiempos más largos (S-105 y S-120), lo que evidenciaría que el tratamiento de post-oxidación no ha sido suficiente para la transformación de estos nitruros en $\mathrm{Fe}_{3} \mathrm{O}_{4}$. La presencia del carburo $\mathrm{Cr}_{23} \mathrm{C}_{6}$ indicaría una cierta sensibilización de este acero a la corrosión intergranular durante el proceso de nitrocarburación (Karl y Beamer, 2016). Al igual que en el acero AISI 4340 , no se encuentra ningún pico de $\mathrm{Fe}_{2} \mathrm{O}_{3}$.

\subsection{Microdurezas}

En la Fig. 4a se muestra de forma gráfica la variación de la dureza del acero AISI 4340 desde la superficie exterior de la capa de óxidos hasta el material base. La dureza se determinó a escala HV 0,1 , excepto la huella 0 que se realizó a escala de $\mathrm{HV}$ 0,025 debido al pequeño espesor de la capa de combinación superficial, $\mathrm{y}$, de esta manera, poder asegurar que únicamente se estaba evaluando esta capa y no la zona de difusión inferior. Se observa un comportamiento similar para todas piezas evaluadas, en las que la dureza disminuye de forma progresiva desde los 950-1150 HV de la zona más externa hasta valores en torno a $350 \mathrm{HV}$ en el material base.

En el acero AISI 347 se encontró una dispersión de valores de dureza considerable (Fig. 4b). Debido a las bajas cargas aplicadas en los ensayos
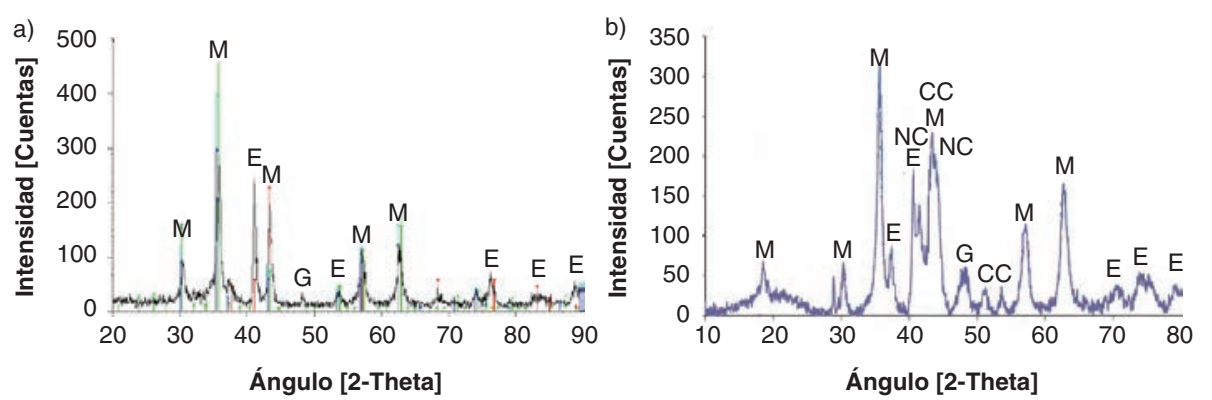

Figura 3. Análisis de difracción de rayos X (XRD) de la superficie de las muestras A-60 (a) y S-60 (b). Símbolos: (M) magnetita; (E) nitruro de hierro $\varepsilon\left(\mathrm{Fe}_{2-3} \mathrm{~N}\right)$; $(\mathrm{G})$ : nitruro de hierro $\gamma^{\prime}\left(\mathrm{Fe}_{4} \mathrm{~N}\right)$; (NC) nitrato de cromo $\left(\mathrm{Cr}_{2} \mathrm{~N}\right)$; CC carburo de cromo $\left(\mathrm{Cr}_{23} \mathrm{C}_{6}\right)$.
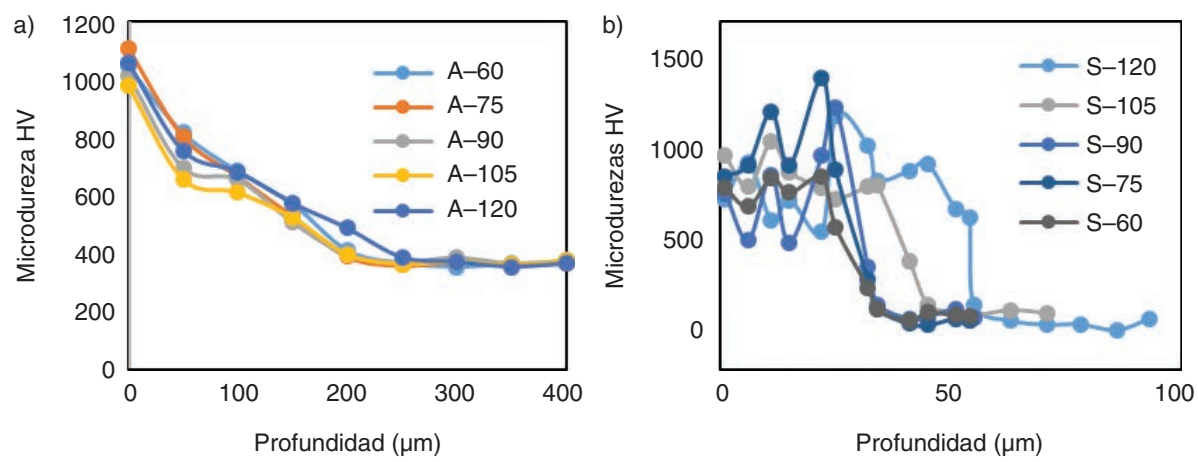

FIGURA 4. Variación de la dureza Vickers con la profundidad en las muestras nitrocarburadas del acero AISI 4340 (a) y del acero AISI 347 (b). 
de nanoindentación, existe una mayor dispersión de valores, siendo más sensibles a la presencia de heterogeneidades de la muestra. En este caso la presencia de nitruros conduce a valores puntuales muy elevados, o muy bajos, dependiendo de cada caso, aunque se observa un comportamiento análogo en todas las muestras evaluadas. Así, se observa una primera zona típica de aceros de aleación relativamente alta en la que los valores de dureza son elevados y dispersos (entre 600 a 1500 HV) para, disminuir marcadamente hasta valores más uniformes (entre 200 y $300 \mathrm{HV}$ ) en el material base. Como era de esperar, la profundidad de comienzo de esta disminución importante de la dureza depende del tiempo de nitrocarburación pasando progresivamente de los $20 \mu \mathrm{m}$ de la muestra S-60 a los $55 \mu \mathrm{m}$ de la muestra S-120. La primera zona cuyo espesor oscila entre $20-50 \mu \mathrm{m}$ es típica de aceros de aleación relativamente alta y se reproduce en los ensayos realizados por otros autores en la nitrocarburación de aceros inoxidables austeníticos (Chang y Chen, 2003).

\subsection{Resistencia al desgaste}

En la Tabla 3 se indican los valores del ratio específico de desgaste, $\mathrm{k}$, en $\mathrm{mm}^{3} \cdot \mathrm{N}^{-1} \cdot \mathrm{m}^{-1}$ obtenidos para las muestras del acero AISI 4340. Dichos valores se han calculado utilizando la siguiente expresión (Archard, 2004):

$\mathrm{k}=\Delta \mathrm{V} / \mathrm{F}_{\mathrm{N}} \mathrm{L}$

siendo $\mathrm{k}$ el coeficiente específico de desgaste $\left(\mathrm{mm}^{3} \cdot \mathrm{N}^{-1} \cdot \mathrm{m}^{-1}\right), \Delta \mathrm{V}$ la pérdida de volumen de la probeta $\left(\mathrm{mm}^{3}\right), F_{\mathrm{N}}$ la fuerza normal aplicada en el contacto $(\mathrm{N})$ y L la distancia de deslizamiento durante el ensayo (m). Se observa que $\mathrm{k}$ es mucho menor en las piezas tratadas mediante el proceso Tenifer$\mathrm{QPQ}{ }^{\circledR}$ que en las no nitrocarburadas debido al aumento de dureza superficial. En el acero AISI 347 se observa que $\mathrm{k}$ es del orden de 20 veces menor en las probetas nitrocarburadas. Dentro de ellas se han alcanzado valores muy semejantes, si bien parece existir una cierta tendencia a aumentar ligeramente el desgaste con el tiempo de tratamiento. En todos los casos, el desgaste ha dado lugar a surcos con una profundidad de aproximadamente 5-6 $\mu \mathrm{m}$ en las probetas tratadas, es decir, que en todos los casos se está ensayando la capa nitrocarburada dado que el óxido es del orden de $2 \mu \mathrm{m}$.

En la Fig. 5a se puede ver la evolución del coeficiente de fricción $\left(\mu_{\mathrm{r}}\right)$ con la distancia de deslizamiento. Se observa que el comportamiento de $\mu_{\mathrm{r}}$ en función del número de ciclos es similar en todas las muestras ensayadas: existe un aumento inicial de $\mu_{\mathrm{r}}$ en la parte inicial de la gráfica hasta alcanzar una zona prácticamente estacionaria, en la que dicho valor oscila entre 0,4 y 0,5 para la muestra A-75 y entre 0,7 y 1,1 para las demás. Este aumento inicial de $\mu_{\mathrm{r}}$ se debe principalmente a la rugosidad superficial, que dificulta el contacto entre el pin de alúmina y la superficie de ensayo, por lo que el área real de contacto es inferior al área aparente de contacto. A medida que aumenta la distancia de deslizamiento también lo hace el área real de contacto y, teniendo en cuenta que ésta y son proporcionales, $\mu_{\mathrm{r}}$ aumenta

TABLA 3. Coeficiente de desgaste $(\mathrm{k})$ y volumen desgastado obtenidos en los ensayos pin-on-disk sobre los aceros AISI 4340 y AISI 347.

\begin{tabular}{lcc}
\hline Muestra & $\begin{array}{c}\text { Coeficiente } \\
\text { de desgaste }(\mathbf{k}) \\
\left(\mathbf{m m}^{\mathbf{3}} \cdot \mathbf{N}^{-1} \cdot \mathbf{m}^{-1}\right)\end{array}$ & $\begin{array}{c}\text { Volumen } \\
\text { desgastado } \\
\left(\mathbf{m m}^{3}\right)\end{array}$ \\
\hline AISI4340 base & $4,41 \cdot 10^{-4}$ & 2,20 \\
A-60 & $1,84 \cdot 10^{-5}$ & 0,09 \\
A-75 & $2,03 \cdot 10^{-5}$ & 0,10 \\
A-90 & $1,86 \cdot 10^{-5}$ & 0,09 \\
A-105 & $1,95 \cdot 10^{-5}$ & 0,09 \\
A-120 & $1,75 \cdot 10^{-5}$ & 0,08 \\
AISI 347 base & $5,44 \cdot 10^{-4}$ & 1,34 \\
S-60 & $8,91 \cdot 10^{-6}$ & 0,02 \\
S-75 & $1,12 \cdot 10^{-5}$ & 0,03 \\
S-90 & $1,08 \cdot 10^{-5}$ & 0,03 \\
S-105 & $1,25 \cdot 10^{-5}$ & 0,03 \\
S-120 & $1,22 \cdot 10^{-5}$ & 0,03
\end{tabular}
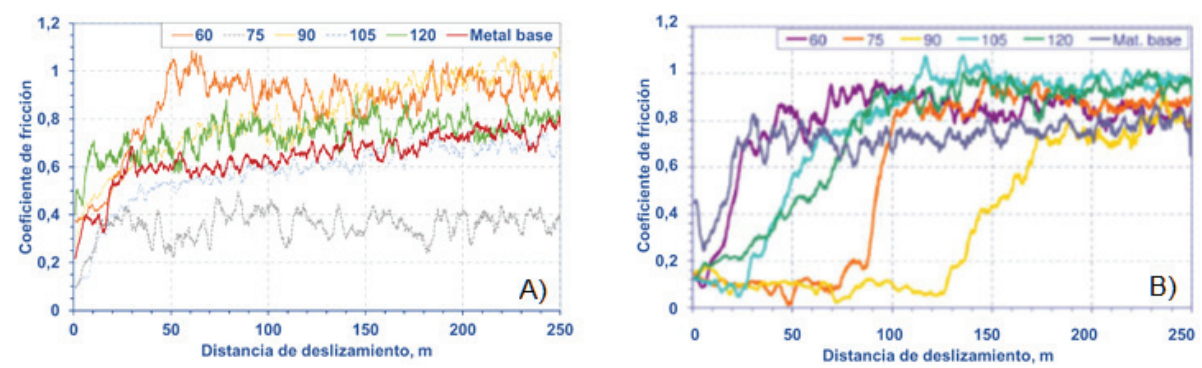

FIGURA 5. Evolución del coeficiente de fricción con la distancia de deslizamiento en el ensayo pin-on-disk para las muestras de acero AISI 4340 (a) y acero AISI 347 (b). 
TABla 4. Análisis de la composición química de dos regiones de apariencia superficial diferente dentro de la huella de desgaste (Espectros 1 y 2) y fuera de ésta (Espectro 3), de la probeta S-60 (\% peso) (ver Fig. 1g)

\begin{tabular}{lccccccccccc}
\hline Espectro & $\mathbf{C}$ & $\mathbf{O}$ & $\mathbf{S i}$ & $\mathbf{C r}$ & $\mathbf{M n}$ & $\mathbf{F e}$ & $\mathbf{N i}$ \\
\hline Espectro 1 & 1,26 & 31,37 & 0,22 & 2,90 & 0,00 & 63,90 & 0,34 \\
Espectro 2 & 1,83 & 30,21 & 0,29 & 10,68 & 0,32 & 51,63 \\
Espectro 3 & 3,77 & 29,11 & 0,60 & 13,11 & 0,58 & 43,44 & 9,90 \\
\hline
\end{tabular}

en la parte inicial del ensayo de forma proporcional a como lo hace el área real de contacto, hasta llegar a la zona estacionaria. Es importante mencionar que el valor de $\mu_{\mathrm{r}}$ obtenido en todas las muestras, a excepción de la A-75, es superior al esperado. Este hecho puede deberse a que la capa superficial obtenida en las piezas A-60, A-90, A-105 y A-120 es más frágil y se rompe en pequeñas partículas que se mantienen en la zona de contacto entre el pin y la pieza, haciendo que el contacto sea irregular y aumente el coeficiente de fricción. Este efecto se observó también al realizar las huellas de dureza, en las que a pesar de utilizar una carga muy baja, se producía la rotura de la capa de óxidos superficial en algunos puntos. En la Fig. 5b se muestra la evolución de $\mu_{\mathrm{r}}$ de las muestras de AISI 347 con la distancia de deslizamiento. Se observa una zona inicial que llega hasta 100 metros, aproximadamente, donde el coeficiente es estable y bajo $(0,1-0,3)$. A partir de aquí, hay que suponer que bien el óxido rompe o bien se produce una interacción con el pin que conduce a un aumento progresivo de $\mu_{\mathrm{r}}$ hasta alcanzar valores de entre $0,6-0,7$ más propios de un contacto tipo metálico, semejante al determinado en el material base. Se realizó un análisis composicional semicuantitativo y morfológico mediante un microscopio electrónico de barrido de las huellas dejadas por el ensayo (Tabla 4). En todas las pruebas se ha encontrado material adherido en forma de parches en el interior del surco. Este material se corresponde, en esencia, con óxido de hierro que presumiblemente procedería del pin de acero (100Cr6) dado el desgaste experimentado por ambas contrapartes, lo que indica que, mientras que la capa de óxido de hierro del acero inoxidable parece estar intacta (salvando ciertos aplastamientos), el pin se ha desgastado considerablemente. Este desgaste implica la generación de partículas que, debido a las temperaturas alcanzadas durante el contacto, se habrían oxidado $\mathrm{y}$, puntualmente, se podrían adherir al material ensayado.

A partir de los resultados obtenidos, la rotura de la capa de óxidos superficiales $\left(\mathrm{Fe}_{3} \mathrm{O}_{4}\right)$ durante el ensayo de desgaste parece indicar que la presencia de esta capa de oxidación no presenta ventajas adicionales en cuanto a la resistencia al desgaste con respecto al tratamiento de nitrocarburación sin etapa de post-oxidación.

\section{CONCLUSIONES}

La principal diferencia microestructural de la capa de nitrocarburación del acero AISI 347 con respecto al AISI 4340 es la no presencia de capa de combinación y la existencia de fases $\gamma^{\prime}-\mathrm{Fe}_{4} \mathrm{~N}$ y $\mathrm{Cr}_{2} \mathrm{~N}$ y $\mathrm{Cr}_{23} \mathrm{C}_{6}$ en el primero de ellos. Este último compuesto indica cierta sensibilización a la corrosión intergranular del acero inoxidable. Sin embargo, el aumento del coeficiente específico de desgaste con respecto $(\mathrm{k})$ con respecto al material de base es similar en ambos casos, adquiriendo valores muy parecidos entre las muestras de cada material con el tiempo de tratamiento, por lo que, con objeto de reducir costes, se aconseja realizar la nitrocarburación de estos aceros por el proceso Tenifer-QPQ ${ }^{\circledR}$ durante $60 \mathrm{~min}$. Por último, indicar que el tratamiento de post-oxidación no presenta ventajas adicionales en cuanto a la resistencia al desgaste.

\section{REFERENCIAS}

Archard, J.F. (2004). Contact and rubbing of flat surface. J. Appl. Phys. 24 (8), 981-988. https://doi.org/10.1063/1.1721448.

Brühl, S.P., Cabo, A., Tuckart, W., Prieto, G. (2016). Tribological behaviour of nitrided and nitrocarburized carbon steel used to produce engine parts. Ind. Lubr. Tribol. 68 (1), 125-133. https://doi.org/10.1108/ILT-07-2015-0101.

Chang, C.N., Chen, F.S. (2003). Wear resistance evaluation of plasma nitrocarburized AISI 316L stainless steel. Mater. Chem. Phys. 82 (2), 281-287. https://doi.org/10.1016/ S0254-0584(03)00234-7.

Dong, H. (2010). S-phase surface engineering of $\mathrm{Fe}-\mathrm{Cr}$, $\mathrm{Co}-\mathrm{Cr}$ and Ni-Cr alloys. Int. Mater. Rev. 55 (2), 65-98. https://doi. org/10.1179/095066009X12572530170589.

Fattah, M., Mahboubi, F. (2010). Comparison of ferritic and austenitic plasma nitriding and nitrocarburizing behavior of AISI 4140 low alloy steel. Mater. Design 31 (8), 39153921. https://doi.org/10.1016/j.matdes.2010.03.008.

Flodström, I. (2012). Nitrocarburizing and High Temperature Nitriding of Steels in Bearing Applications. Master of Science Thesis, Department of Materials and Manufacturing Technology, Chalmers University of Technology, Göteborg, Sweden.

Funatani, K. (2004). Low-Temperature Salt Bath Nitriding of Steels. Met. Sci. Heat Treat. 46 (7-8), 277-281. https://doi. org/10.1023/B:MSAT.0000048834.48163.2e.

Karl, A., Beamer, C. (2016). Applications and Design of Low Temperature Surface Hardened Stainless Steel Components in Automotive Applications. SAE Int. J. Mater. Manuf. 9 (3), 679-684. https://doi.org/10.4271/2016-01-0425.

García, A., Vargas, G., López, J. (2018). Surface microstructural evolution of AISI $304 \mathrm{~L}$ stainless steel oxy-nitrocarburized in a cyanide-free salt bath and its potential application in solar collectors. Surf. Coat. Tech. 353, 190-198. https://doi. org/10.1016/j.surfcoat.2018.08.078. 
Li, G., Peng, Q., Li, C., Wang, Y., Gao, J., Chen, S., Wang, J., Shen, B. (2008a). Microstructure analysis of $304 \mathrm{~L}$ austenitic stainless steel by QPQ complex salt bath treatment. Mater. Charact. 59 (9), 1359-1363. https://doi. org/10.1016/j.matchar.2007.09.011.

Li, G., Wang, J., Peng, Q., Li, C., Wang, Y., Shen, B. (2008b). Influence of salt bath nitrocarburizing and post-oxidation process on surface microstructure evolution of $17-4 \mathrm{PH}$ stainless steel. J. Mater. Process. Tech. 207, 187-192. https://doi.org/10.1016/j.jmatprotec.2007.12.082.

Marušić, K., Otmačić, H., Landek, D., Cajner, F., StupnišekLisac, E. (2006). Modification of carbon steel surface by the Tenifer $\AA$ process of nitrocarburizing and post-oxidation. Surf. Coat. Tech. 201 (6), 3415-3421. https://doi. org/10.1016/j.surfcoat.2006.07.231.

Mittemeijer, E.J. (2013). Fundamentals of Nitriding and Nitrocarburizing. In Steel Heat Treating Fundamentals and
Processe. Dossett, J. and Totten, G.E (Editors), Volume 4A, ASM Handbook, ASM International, Materials Park (Ohio), pp. 619-646.

Schneider, R.S.E. (2014). Austenitic nitriding and nitrocarburizing of steels. In Thermochemical Surface Engineering of Steels: Improving Materials Performance. Mittemeijer, E.J., Somers, M.A.J (Eds.), Elsevier, Amsterdam, pp. $373-400$.

Wang, J., Lin, Y., Zhang, Q, Zeng, D., Fan, H. (2014). Effect of treatment time on the microstructure of austenitic stainless steel during low-temperature liquid nitrocarburizing. Metall. Mater. Trans. A 45 (10), 4525-4534. https://doi. org/10.1007/s11661-014-2418-7.

Wu, D., Ge, Y., Kahn, H., Ernst, F., Heuer, A.H. (2015). Diffusion profiles after nitrocarburizing austenitic stainless steel. Surf. Coat. Tech. 279, 180-185. https://doi.org/10.1016/j. surfcoat.2015.08.048 